\title{
The small ubiquitin-like modifier (SUMO) is required for gonadal and uterine-vulval morphogenesis in Caenorhabditis elegans
}

\author{
Limor Broday, ${ }^{1,2,5}$ Irina Kolotuev, ${ }^{2}$ Christine Didier, ${ }^{1}$ Anindita Bhoumik, ${ }^{1}$ Bhagwati P. Gupta, ${ }^{3,4}$ \\ Paul W. Sternberg, ${ }^{3}$ Benjamin Podbilewicz, ${ }^{2}$ and Ze'ev Ronai ${ }^{1,6}$ \\ ${ }^{1}$ Department of Oncological Sciences, Mount Sinai School of Medicine, New York, New York 10029, USA; ${ }^{2}$ Department of \\ Biology, Technion-Israel Institute of Technology, Haifa 32000, Israel; ${ }^{3}$ Howard Hughes Medical Institute and Division of \\ Biology, California Institute of Technology, Pasadena, California 91125, USA
}

The small ubiquitin-like modifier (SUMO) modification alters the subcellular distribution and function of its substrates. Here we show the major role of SUMO during the development of the Caenorhabditis elegans reproductive system. smo-1 deletion mutants develop into sterile adults with abnormal somatic gonad, germ line, and vulva. SMO-1:: GFP reporter is highly expressed in the somatic reproductive system. smo-1 animals lack a vulval-uterine connection as a result of impaired ventral uterine $\pi$-cell differentiation and anchor cell fusion. Mutations in the LIN-11 LIM domain transcription factor lead to a uterine phenotype that resembles the smo-1 phenotype. LIN-11 is sumoylated, and its sumoylation is required for its activity during uterine morphogenesis. Expression of a SUMO-modified LIN-11 in the smo-1 background partially rescued $\pi$-cell differentiation and retained LIN-11 in nuclear bodies. Thus, our results identify the reproductive system as the major SUMO target during postembryonic development and highlight LIN-11 as a physiological substrate whose sumoylation is associated with the formation of a functional vulval-uterine connection.

[Keywords: SUMO; somatic gonad; smo-1; lin-11; uterine-vulval connection]

Supplemental material is available at http://www.genesdev.org.

Received May 28, 2004; revised version accepted August 9, 2004.

The small ubiquitin-like modifier (SUMO) is a member of the ubiquitin-like superfamily, which consists of three family members in vertebrates (SUMO1-3) (for review, see Melchoir 2000; Alarcon-Vargas and Ronai 2002; Müller et al. 2004) and a single member (SMT3) in invertebrates (Schwarz et al. 1998). SUMO conjugation has been shown to affect subcellular localization of the modified substrate, thereby affecting its activity and stability (Matunis et al. 1996; Mahajan et al. 1997; Muller at al. 1998). Several transcription factors are modified by sumoylation. Whereas SUMO modification negatively regulates the androgen receptor, SP3, c-Jun, and p53 (Gostissa et al. 1999; Muller et al. 2000; Poukka et al. 2000; Schmidt and Muller 2002), sumoylation of the glucocorticoid receptor increases its transcriptional activi-

\footnotetext{
${ }^{4}$ Present address: Department of Biology, McMaster University, Hamilton, Ontario L8S 4K1, Canada. Corresponding authors.

${ }^{5}$ E-MAIL limor.broday@mssm.edu; FAX (212) 849-2425.

${ }^{6}$ E-MAIL zeev.ronai@mssm.edu; FAX (212) 849-2425.

Article and publication are at http://www.genesdev.org/cgi/doi/10.1101/ gad.1227104
}

ties (LeDrean et al. 2002). Sumoylation also affects transcriptional activities indirectly. For example, SUMO conjugation to class II histone deacetylase impairs its transcription-repressing function (Kirsch et al. 2002). Alternatively, sumoylation has also been shown to affect nuclear and subnuclear (nucleolar or PML nuclear body) localization of regulatory proteins primarily implicated in transcriptional control (Sternsdorf et al. 1997; Pichler et al. 2002).

The SUMO conjugation system is essential for viability in Saccharomyces cerevisiae (Melchoir 2000). Phenotypes observed upon aberrant sumoylation in $S$. cerevisiae include impaired septin ring formation, chromosomal segregation, and progression of the cell cycle through $\mathrm{G}_{2}-\mathrm{M}$ (Johnson and Blobel 1999). Studies in Arabidopsis suggest that the SUMO conjugation system has a role in protection against stress and/or repair of stressrelated damage (Kurepa et al. 2002). In Drosophila melanogaster, the loss-of-function mutation of semushi, the UBC9 (SUMO-conjugating enzyme) ortholog, prevents nuclear import of the transcription factor Bicoid (Bcd) and results in impaired embryogenesis (Epps and Tanda 1998). 
The Caenorhabditis elegans SUMO (smo-1) has been cloned (Choudhury and Li 1997) and predicted to be a single gene (K12C11.2) encoding for a 91-amino acid protein (Coulson 1996; The C. elegans Sequencing Consortium 1998). smo-1 RNAi has been found to cause embryonic arrest in $100 \%$ of progeny, implying that SUMO is required for normal embryogenesis in C. elegans (Fraser et al. 2000; Jones et al. 2002). Recently, it was shown that sumoylation of the C. elegans Polycomb group (PcG) protein, SOP-2, is required for its activity (Zhang et al. 2004).

To elucidate the nature of SUMO modification in $C$. elegans, we characterized the changes in the development of a smo-1 deletion mutant and identified LIN-11, a LIM-homeodomain transcription factor (Ferguson and Horvitz 1985; Freyd et al. 1990; Newman et al. 1999), as a substrate for sumoylation.

\section{Results}

\section{Characterization of smo-1 loss-of-function mutant}

Deletion of the smo-1 gene (Fig. 1A-C) enabled characterization of the SUMO conjugation system during $C$. elegans development. The smo-1(ok359) allele is recessive and fully penetrant for sterility. Earlier studies revealed that smo-1(RNAi) causes $100 \%$ penetrant embryonic lethality (Fraser et al. 2000; Jones et al. 2002; L. Broday and Z. Ronai, unpubl.). Such lethality was not observed in the deletion homozygous derived from smo-1/+ heterozygous, probably because of maternally contributed smo-1(+) product. Therefore, the RNAi results suggest that the null phenotype of smo-1 is embryonic lethal and that in smo-1(ok359) heterozygous animals there is maternal rescue of the embryonic lethality but not the sterility. At $20^{\circ} \mathrm{C}$, smo-1(ok359) embryos hatch and develop into sterile adults with aberrant somatic gonad, germ line, and vulva (Fig. 1E,F). Rescue experiments by germ-line transformation with a genomic construct spanning the smo-1 region (Fig. 1D) show that the phenotypes observed are due to the smo-1 deletion allele and not to closely linked additional mutations, and that the smo-1(ok359) allele is recessive with respect to the protruding vulva $(\mathrm{Pvl})$ and sterile (Ste) phenotypes.

\section{Aberrant somatic gonad and vulva in smo-1 animals}

The somatic gonad of the smo-1(ok359) mutant was analyzed using the AJM-1 reporter (Francis and Waterson 1991; Podbilewicz and White 1994; Köppen et al. 2001). Staining with the monoclonal antibody MH27 (recognizing the AJM-1 protein), and analysis of the ajm-1::GFP reporter in combination with Nomarski optics observations revealed that the uterine, vulval, and spermathecal cells are present in smo-1(ok359) animals, but fail to form normal structures (Figs. 1F, 2A-F). An abnormal distribution of antigen indicative of disorganized structures of the spermathecal cells was observed in adult animals. In addition, the spermatheca (sp) lacked fully
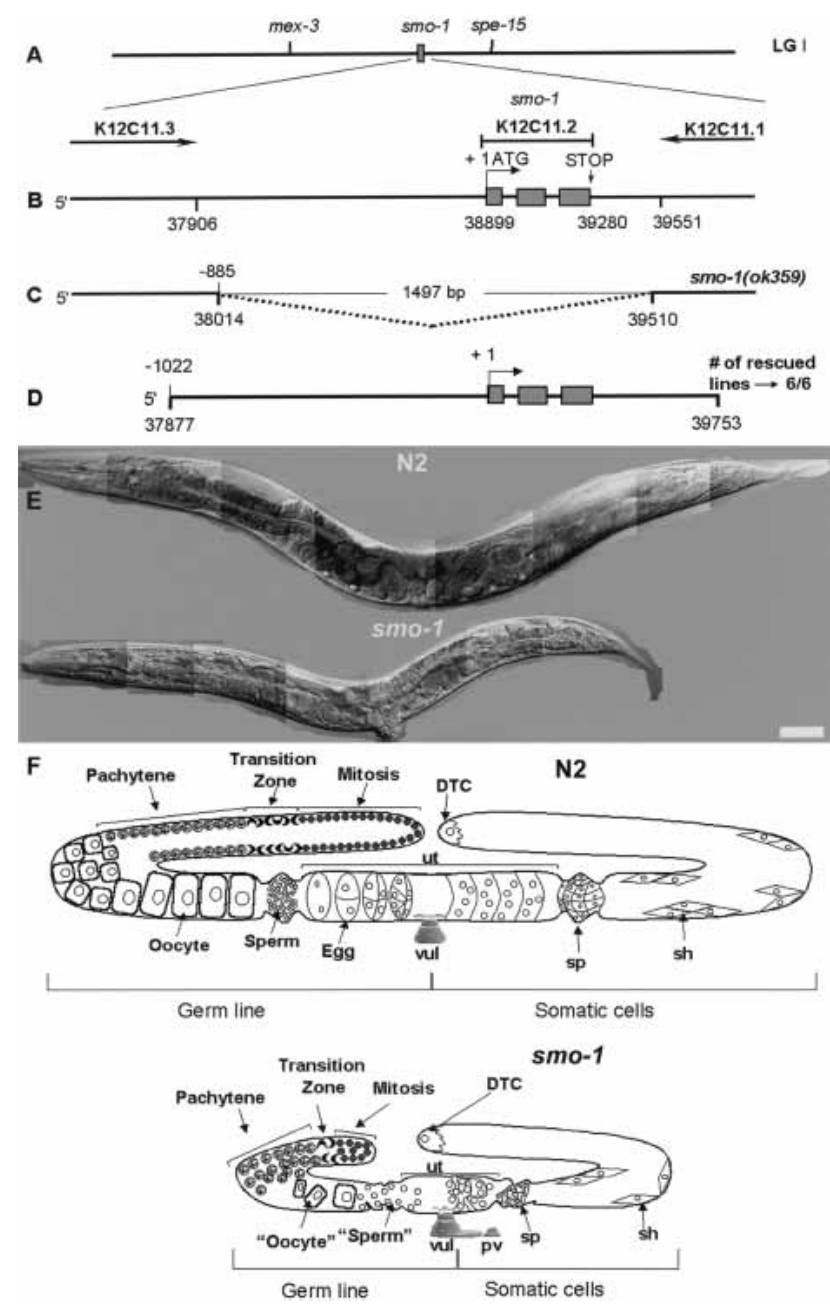

Figure 1. smo-1 genomic region, deletion mutant, and rescue construct. (A) Genetic map position of smo-1. (B) The genomic structure of smo-1. Exons are depicted as shaded boxes. Above is the organization of the $\mathrm{K} 12 \mathrm{C} 11$ cosmid and the nearby predicted genes. Coordinates refer to base numbers on cosmid K12C11. (C) The genomic structure of the smo-1(ok359) deletion mutant. $(D)$ The genomic construct used to rescue the smo1(ok359) mutant animals. (E) Nomarski photomicrographs of wild-type and smo-1(ok359) adult homozygous animals. The mutant adult is smaller with protruding vulva (Pvl) and aberrant germ line. Bar, $50 \mu \mathrm{m}$. (F) Schematic representation of the adult gonad and vulva in wild-type and smo-1 null mutants.

differentiated sperm (Fig. 2A,B). The mutant animals lacked the uterine toroids or exhibited impaired cellular morphogenesis (Fig. 2, cf. A,D and B,F), and the uterine lumen could not be detected at the L4 stage using Nomarski optics (Fig. 2, cf. C and E). These observations provide evidence for the essential role of the sumoylation process in the normal development of the spermatheca and uterus.

Analysis of the vulva in smo-1(ok359) homozygous animals revealed high variability in the organization and shape of the final vulval structures $(n>200)$. Although vulval invagination appears normal (Sharma-Kishore et al. 1999), later migration of vulA-D cells was often slow 


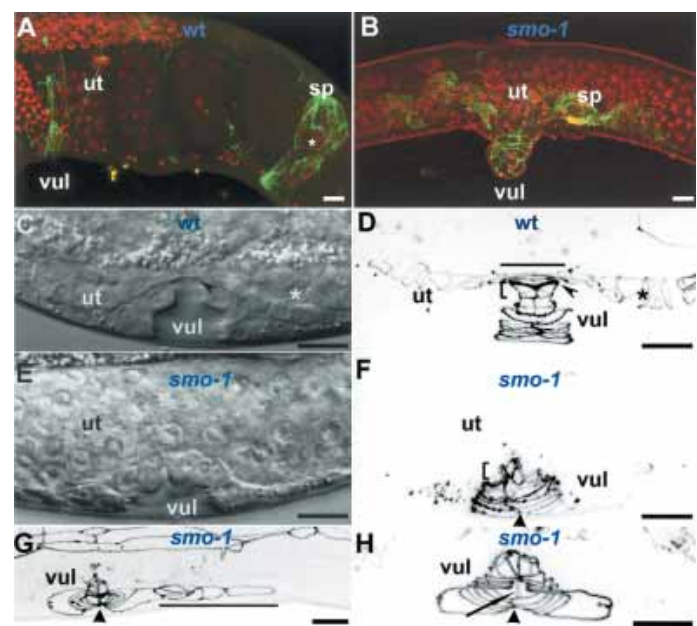

Figure 2. Analysis of the somatic gonad and vulva of the smo1(ok359) animals using the AJM-1 reporter. Wild-type $(A)$ and smo-1(ok359) (B) adult hermaphrodites stained with the MH27 antibody (green). Nuclei are stained with PI (red). Disorganization of the uterine toroids and spermathecal cells is shown in $B$. (C) Nomarski photomicrograph of wild-type mid-L4-stage animal. Arrowhead indicates the utse cell cytoplasm. Asterisk indicates the uterine lumen. $(D)$ Wild-type expression pattern of the ajm-1::GFP reporter. Line indicates the utse; arrowhead indicates the adherens junctions between uv1 cells and VulF; bracket indicates VulF; asterisk indicates the uterine lumen. $(E)$ Nomarski photomicrograph of a smo-1(ok359) mid-L4 animal with aberrant uterus and vulval ring formation. No uterine lumen and uterine-vulval connection could be detected. $(F-H)$ Expression pattern of the ajm-1::GFP in smo-1(ok359) mid-L4 animals. (F) A bracket indicates aberrant VulF. The uterine cells (see $E$ ) do not form the uterine toroids and therefore do not express the ajm-1::GFP reporter. $(F, G, H)$ Arrowheads indicate migration defects of vulval cells. $(G)$ Ectopic posterior vulva is indicated by black line. $(H)$ Migration defects of the vulval cells (arrowhead) that prevent normal ring formation. Arrow indicates the two VulF cells that did not attach properly. (ut) Uterus; (vul) vulva; (sp) spermatheca. Bars, $10 \mu \mathrm{m}$.

or arrested but usually was completed in a retarded way at the late L4 stage (see arrowheads in Fig. 2F-H). Inhibition of cells' attachment (arrow in Fig. 2H) and a block in intratoroidal cell fusions was also observed. The final structures of vulE and vulF cells were aberrant in $19 \%$ $(n=32)$ of the animals (Fig. 2, cf. brackets in D and F). Ectopic vulval differentiation could be detected in $31 \%$ $(n=32)$ of the animals (Fig. 2G). Interestingly, the occurrence of pseudo-vulva structures was restricted to the posterior side of the real vulva (although at low frequencies, the anterior P4.p escaped fusion but did not form pseudo-vulva). The unfused cells that form the posterior ectopic vulva appear to develop from the P8.p cell that escaped fusion to the hypodermis and then proliferated followed by cell migrations.

All homozygous smo-1(ok359) adults $(n>1000)$ had a Pvl (Figs. 1E, 2B). The Pvl phenotype could be attributed to both the presence of an abnormal vulE cell, which is expected to connect to the seam cells by the vulva muscles, and impaired uterine-seam cell (utse) formation (see below). smo-1 is required for germ-line development

Analysis of gonad morphology in wild-type and smo1(ok359) young adults revealed gonad migration defects in $41 \%(22 / 54)$ of the mutant hermaphrodites. Impaired migration could be mainly attributed to failure of the gonadal distal tip cells (DTCs) to migrate dorsally, which halted migration before the first turn (Fig. 3, cf. A and C). In addition, the gonad arms of the mutant animals were smaller than those of wild-type gonads (Figs. 1F, 3A-C).

One-hundred percent of the smo-1(ok359) homozy-
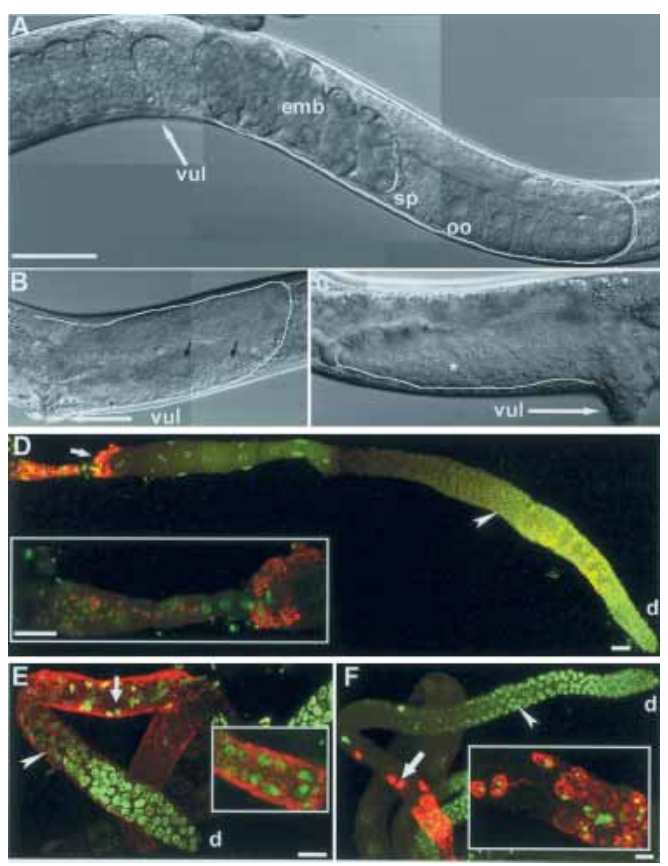

Figure 3. Somatic gonad and germ line in wild-type and smo1(ok359) adult hermaphrodite. $(A-C)$ Nomarski photomicrographs of wild-type and smo-1(ok359) hermaphrodites. One gonadal arm is shown for each hermaphrodite. (A) Wild-type adult gonad arm. Sperm is stored in the spermatheca (sp). The proximal germ line contains the oocytes (oo), and the uterus contains developing embryos (emb). Line along the gonad indicates gonad migration patterns. $(B, C)$ smo-1(ok359) germ lines. $(B)$ Gonad is small and misshapen, with two defective oocytes (black arrows indicate oocyte nuclei). (C) Severe gonad migration defect (indicated by the line) and accumulation of defective gametes throughout the gonad arm (labeled with $\left.{ }^{\star}\right) .(A-C)$ (vul) Vulva. Anterior is to the left of the page. $(D-F)$ Sperm differentiation in wild-type and smo-1(ok359) germ lines. Dissected gonads were stained with the spermatogenic-specific antibody SP56 (red). Nuclei were stained with SYTO 24 (green). (d) Distal gonad. (D) Wild-type sperm are compact and the staining is localized to the proximal germ line. $(E, F)$ Defective spermatogenic cells in smo1(ok359) adult germ line. (E) Defective spermatogenic cells are larger relative to mature sperm and resemble primary spermatocytes that did not differentiate further (arrow indicates sperm nucleus). (F) The labeled sperm cells are more compact (arrow) relative to those in $E$ and resemble secondary spermatocytes. No mature sperm is observed, and there are less sperm cells than in the wild-type germ line. Note the aberrant and noncontinuous SP56 staining pattern. Insets in $D-F$ show magnification of sperm. Bar, $10 \mu \mathrm{m}$. 
gous animals were completely sterile and did not form normal gametes. Neither mature sperm nor normal oocytes could be found in the adult mutants (Fig. 3B,C). The degree of aberrant sperm and oocyte formation varied from germ lines that contained only aberrant spermatogenic cells to those that also contained several defective oocytes. Although germ cells entered the meiotic prophase, as revealed by the presence of pachytene-stage meiotic germ cells (SYTO, green DNA staining, arrowheads in Fig. 3D-F), they failed to form both normal sperm and oocytes. Adult sperm structure and localization within the germ line were analyzed by staining with the SP56 antibody (Ward et al. 1986) (red staining, arrows in Fig. 3D-F). Staining revealed that the structure of the spermatogenic cells in the smo-1(ok359) germ lines is defective and that sperm localization is not restricted to the proximal gonad as in the wild type (Fig. 3, cf. D and $\mathrm{E})$.

\section{SMO-1::GFP expression in the somatic gonad}

To examine the expression pattern of smo-1, we generated a full-length SMO-1::GFP translational fusion construct. Three stable lines containing an extrachromosomal array of the translational fusion that exhibited stable expression patterns were examined (Fig. 4). At the L4 stage, SMO-1::GFP marker was expressed in each of the 64 uterine cells (Fig. 4A-D), the somatic spermathecal cells, the sheath cells that cover the gonadal arms (Fig. 4E,F), the hypodermis, and seam cells (Fig. 4B). The two DTCs of the somatic gonad also expressed the transgene (Fig. 4D). At the early L3 stage, expression was detected in the six vulval precurcor cells (VPCs) (Supplementary Fig. S1), and at the L4 stage SMO-1::GFP was found in all 22 vulval nuclei (Fig. 4B,C), as well as in the gonadal anchor cell (AC) (Fig. 4C, inset). The lack of germ cell expression could be attributed to germ-line silencing (Kelly and Fire 1998). Expression in embryos (Supplementary Fig. S2) was detected as early as the gastrulation stage.

The expression of the SMO-1::GFP construct in the somatic gonad together with the sterility and abnormal gonad morphology in smo-1 mutants suggests that the SUMO modification system is essential for normal formation of the somatic gonad. Expression of the SMO$1::$ GFP reporter was also detected in the ventral nerve cord (vnc), in the nerve ring, and in head and tail neurons (Fig. 4B), indicating that SUMO may function in the nervous system. We could not detect expression above background levels in the intestine and in body-wall muscles.

\section{Aberrant uterus-vulva connection in smo-1 animals}

Among the smo-1(ok359) mutant phenotypes is the Cog (connection of gonad defective) phenotype. The Cog phenotype could be a result of impaired generation of utse, a failure of vulva attachment to the utse, or a failure in fusion between the anchor cells (ACs) and the utse (Hanna-Rose and Han 1999). The uterine $\pi$ cells generate cells of two types that connect to the vulva:
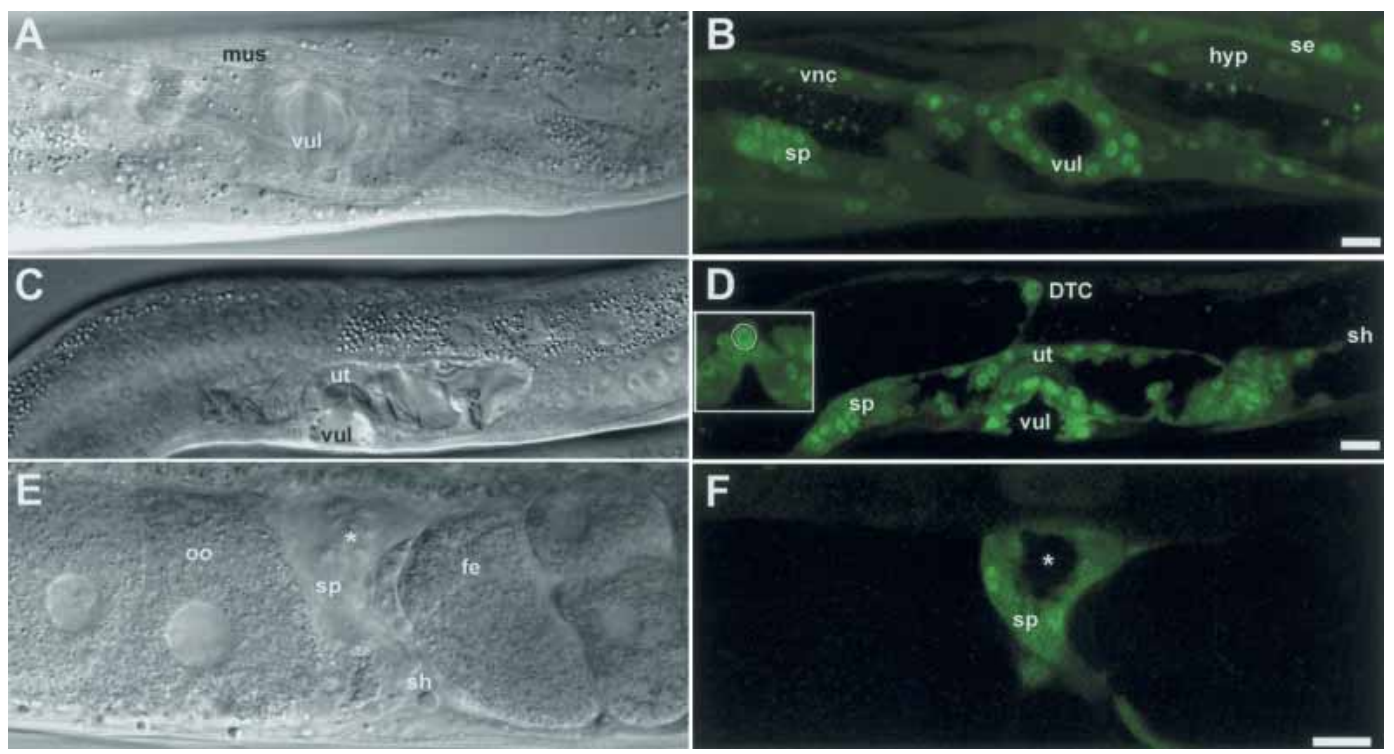

Figure 4. smo-1::GFP is expressed in the entire somatic gonad. Nomarski photomicrographs $(A, C, E)$ of wild-type animals expressing the smo-1::GFP reporter and corresponding fluorescence images $(B, D, F) .(A, B)$ Ventral view of mid-L4 animal. Expression is detected in all vulval cells (vul), spermathecal cells (sp), hypodermis (hyp), seam cells (se), and ventral nerve cord (vnc). (C,D) Lateral view of mid-L4 animal. Expression is detected in all vulval cells (vul), spermathecal cells (sp), sheath cells (sh), all uterine cells (ut), and the distal tip cell (DTC). Inset shows transgene expression in the anchor cell of earlier-stage animals. $(E, F)$. Lateral view of adult spermatheca. Expression is restricted to the somatic spermathecal cells (sp) and sheath cells (sh). There is no expression in sperm (*), oocytes (oo), fertilized egg (fe), or two-cell embryos (right of the fertilized egg). Expression in embryos was detected from the gastrulation stage (see Supplemental Material). Bar, $10 \mu \mathrm{m}$. 
Broday et al.

utse and uv1. The utse is a multinucleate cell that forms a thin laminar process at the uterus-vulva interface. The uvl cells form adherens junctions with vulF and with the utse (Newman and Sternberg 1996; Newman et al. 1996). These junctions are clearly stained with the AJM-1 reporter in wild-type but not in smo-1(ok359) animals, demonstrating the lack of the uterine-vulval connection in the mutant (Fig. 2, cf. C,D and $\mathrm{E}, \mathrm{F})$.

In wild-type animals, in addition to vulval induction, the AC induces $\pi$-cell fate. The AC then fuses to eight of the $\pi$-cell progeny to form the utse (Newman et al. 1995, 1996; Newman and Sternberg 1996). The AC does not appear to be aberrant in its morphology during the L3 stage in smo-1(ok359) homozygous hermaphrodites. However, the mutant animals lack the utse and the AC remains unfused until the late L4 stage, exhibiting an abnormal morphology of a bloated cell above the vulva apex (Fig. 5C,E). In wild-type animals, the GFP fluorescence of a $z m p-1:: \mathrm{GFP}$ reporter (Wang and Sternberg 2000) spreads from the AC cytoplasm to the utse upon fusion of the AC with the multinucleate utse, resulting in a diffuse labeling (Fig. 5, cf. A,B and C,D). The zmp$1::$ GFP expression in the AC of smo-1(ok359) animals remained distinct until the late L4 stage, providing additional support for lack of fusion of the AC in the absence of SUMO (Fig. 5E,F).

To study the fate of the uterine $\pi$ cells in smo- 1 animals, we used a $\operatorname{cog}-2:$ :GFP reporter (Hanna-Rose and Han 1999|. We found that $\operatorname{cog}-2::$ GFP is visible in the $\pi$ cells of smo-1(ok359) animals during early L4, but its expression is not restricted to the $\pi$ cells, and additional uterine cells express the marker with lower intensity (Fig. 5, cf. G,H and I,J). During the late L4 stage, the $\operatorname{cog}-2::$ GFP-expressing cells are spread randomly in the uterus and are labeled at various intensities [Fig. 5K,L; $n=20$ for wild-type and $n=24$ for smo-1(ok359) animals]. Impaired $\pi$-cell specification in addition to the absence of AC fusion (resulting from the lack of utse) may underlie the aberrant differentiation of the utse and uv1 cells and may prevent formation of the vulva-uterus connection (Fig. 5M).

Figure 5. $(A-F)$ Expression of the $z m p-1:$ GFP reporter in the anchor cell (AC) of smo-1(ok359) animals demonstrates that the AC fails to fuse. Nomarski photomicrographs $(A, C, E)$ and corresponding fluorescence images $(B, D, F)$, lateral views. $(A, B)$ Wild-type mid-L4 stage vulva after the AC fusion to the utse cell, showing diffusion of the zmp-1::GFP reporter throughout the multinucleate utse cell. $(C, D)$ smo-1(ok359) animal at midL4 stage. $(E, F)$ smo-1(ok359) animal at late L4 stage. GFP expression is restricted to the $\mathrm{AC}$ even at the late L4 stage, as the AC remains unfused. Arrow indicates additional expression of the zmp-1::GFP reporter in vulval cells. (vul) Vulva; (AC) anchor cell. Bar, $10 \mu \mathrm{m}$. $(G-M)$ Expression of the $\operatorname{cog}-2::$ GFP reporter in smo1(ok359) animals demonstrates aberrations in $\pi$-cell differentiation. Nomarski photomicrographs $(G, I, K)$ and corresponding fluorescence images $(H, J, L)$, lateral views. $(G, H)$ A wild-type mid-L4 stage vulva. The $12 \pi$ cells, six cells from each side of the uterus (one side is shown) migrate anteriorly and posteriorly to form the utse and uvl cells. Asterisk indicates a $\pi$-cell nucleus. $(I, J)$ A smo-1(ok359) animal at mid-L4 stage. Additional uterine cells expressing the $\operatorname{cog}-2:: \mathrm{GFP}$ reporter with weaker intensity are indicated by arrows. $(K, L) \mathrm{A}$ smo1(ok359) animal at late L4 stage. The $\pi$ cells migrate randomly and additional uterine cells are labeled (arrows). (m) Muscle cell; (ut) uterine lumen; (vul) vulva. Bar, $10 \mu \mathrm{m}$. (M) Schematic comparison of the development of the uterine-vulval connection at the L4 stage in wild-type and smo-1 null mutants. Aberrations in $\pi$-cell differentiation and migration and ectopic random expression of a specific $\pi$-cell marker $(\operatorname{cog}-2:: \mathrm{GFP})$ in additional uterine cells prevent the normal formation of the uterine-vulval connection in smo-1 null animals.
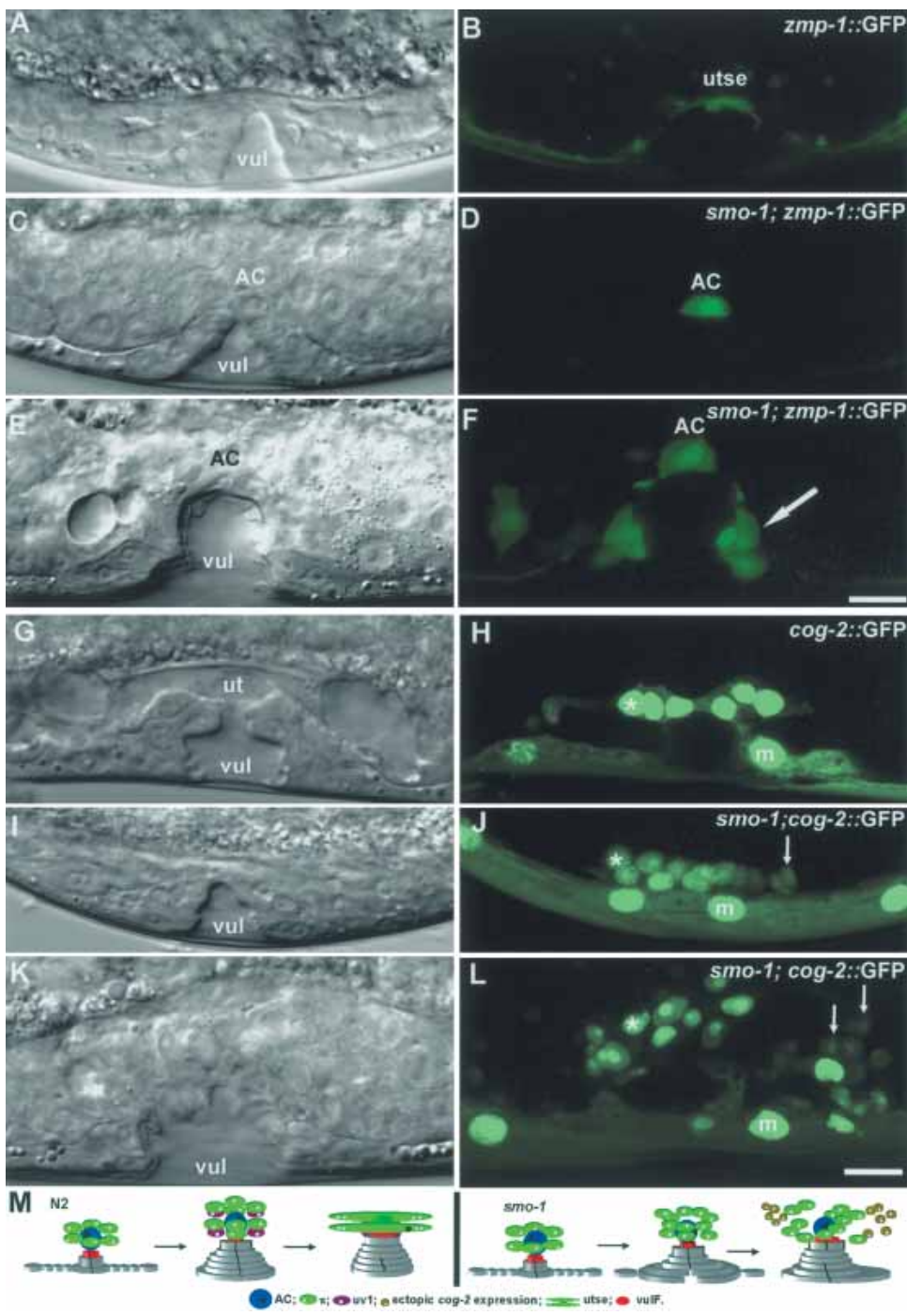


\section{SUMO modification of LIN-11 affects its function and nuclear localization}

lin-11(n389) mutant animals lack the vulval-uterine connection and the AC remains on the top of the vulva apex, resulting in its failure to fuse and migrate (Newman et al. 1999). In addition, the expression pattern of the $\operatorname{cog}-2::$ GFP reporter in lin-11(n389) background is similar to that detected in smo-1(ok359) animals (Supplementary Fig. S3) and indicates impaired $\pi$-cell differentiation. These observations prompted us to examine the possibility that LIN-11 is one of the SUMO substrates and that sumoylation of LIN-11 may be required for its normal expression and function. For this purpose, we analyzed the expression pattern of the LIN$11:$ GFP translational fusion construct (plin-11ABCDE::GFP, which does not include the LIN-11 homeobox domain) (Hobert et al. 1998). This construct is expressed in wild-type animals both in the nucleus and in the cytoplasm of the $\pi$ cells and vulval cells (Fig. 6A,B; $n=22$ ). In the smo-1(ok359) uterus, the $\pi$ cells are not normally differentiated as shown above (Fig. 5J,L). The intensity of the LIN-11::GFP transgene in smo-1 (ok359) was much weaker and diffused compared with the wildtype pattern (Fig. 6C,D; early L4 $n=23 / 26$ ) and could be detected with high variability only in a few uterine cells (Fig. 6E,F; late L4) (one to four cells in each side of the uterus, compared with six cells in the wild type). In a few cases the transgene was not expressed at all in the uterus $(n=3 / 26)$. Moreover, whereas in the wild-type developing uterus the $\pi$ cells migrate after dividing, in the smo1 (ok359) animals the LIN-11::GFP-expressing cells were clustered and failed to occupy the correct stereotypic positions (Fig. 6, cf. D and B; also cf. Fig. $5 \mathrm{H}, \mathrm{J}$ ). Based on this observation we hypothesized that sumoylation of LIN11 is involved in its uterine function. To further elucidate this possibility, we established three transgenic lines which express plin-11::MYC::SMO-1 ::LIN-11 translational fusion in the smo-1(ok359) genetic background (see Materials and Methods). This construct may mimic sumoylation of LIN-11 as was shown for Sp3 and huntingtin (Ross et al. 2002; Steffan et al. 2004). It was demonstrated that covalent attachment of SUMO to Sp3 regulated its activity as a transcriptional repressor, independent of the position of SUMO attachment (Ross et al. 2002). Immunostaining of the transgenic plin$11::$ MYC::SMO-1::LIN-11 animals with MH27 and anti-myc antibodies revealed that defects in late stages of vulval development, especially in cell migration events, are more severe relative to the nontransgenic smo1 (ok359) vulvae. However, the uterine $\pi$ cells are better differentiated in the plin-11::MYC::SMO-1::LIN-11 transgenic smo-1(ok359) animals. Six $\pi$ cells that are normally positioned in each side of the uterus (in all, 12 cells) could be detected (Fig. 6G; $n=15 / 20$ ). In addition, the plin-11::MYC::SMO-1::LIN-11 fusion protein is localized to distinct nuclear bodies. Transgene expression could not be detected in smo-1(ok359) homozygous animals harboring the control construct plin$11::$ MYC $::$ LIN-11 ( $n=3$ independent lines; $n>100)$ but

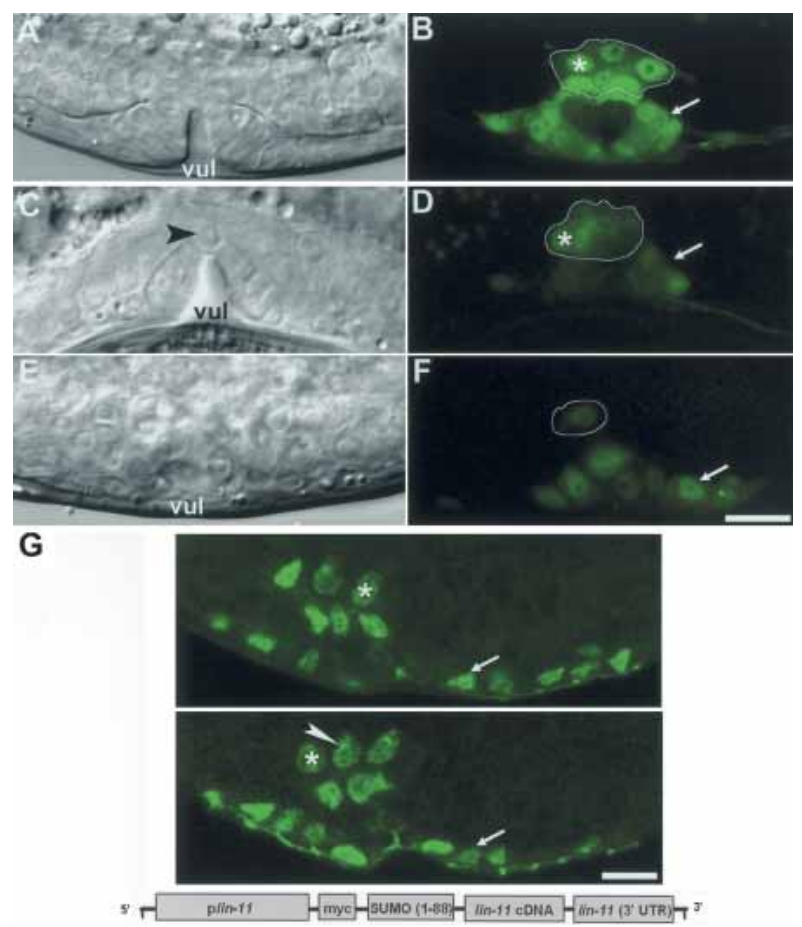

Figure 6. LIN-11 protein expression and localization are regulated by SUMO modification. $(A-F)$ Expression of the lin$11::$ GFP reporter in the $\pi$ cells of wild-type and smo-1(ok359) animals. Nomarski photomicrographs $(A, C, E)$ and corresponding fluorescence images $(B, D, F)$, lateral views. $(A, B)$ A wild-type mid- L4 stage vulva. GFP expression is shown in the $\pi$ cells $\left(^{\star}\right)$ and secondary-fate vulval cells (arrow). The six $\pi$ cells shown are arranged in two lines (outline). (C,D) A smo-1(ok359) animal at mid-L4 stage. $(C)$ The unfused anchor cell is indicated by an arrowhead. (D) GFP expression is weaker and nuclear labeling decreased relative to the cytoplasmic labeling. The $\pi$ cells are clustered (outline). (E,F) A smo-1(ok359) animal at late L4 stage. Weak expression is detected only in one uterine $\pi$ cell (outline). $(G)$ Immunofluorescence staining with MH27 and the anti-myc antibodies of a transgenic smo-1(ok359) animal expressing the plin-11::MYC::SUMO::LIN-11 fusion protein. The upper and lower panels show the two sides of the uterus. Note six $\pi$ cells in each side of the uterus (star). LIN-11 is expressed in discrete nuclear dots (arrowhead). Arrow indicates one of the vulval cells. Bar, $10 \mu \mathrm{m}$.

was detected in wild-type or heterozygous animals that contains endogenous SUMO (data not shown), and was also functional in rescuing the lin-11(n389) mutant (see below, Fig. 7D). Together, these data indicate that covalent attachment of SUMO to LIN-11 increases the level and nuclear retention of LIN-11 and target LIN-11 to discrete nuclear dots. Whereas sumoylation of LIN-11 partially rescued the differentiation of the uterine $\pi$ cells in the smo-1(ok359) mutant, SUMO-modified LIN-11 further impaired late stages of vulval development.

To confirm direct sumoylation of LIN-11, we transfected myc-LIN-11 and HA-SUMO to 293T cells. Immunoprecipitation of LIN-11 followed by immunoblot with antibodies to SUMO confirmed sumoylation of LIN-11 in mammalian cells. Furthermore, SUMO antibodies 
Broday et al.

Figure 7. $\mathrm{K} 17$ and $\mathrm{K} 18$ are the major SUMO acceptor sites in LIN-11. $(A$, left $)$ LIN-11 is modified by SUMO in mammalian cells. 293T cells were transfected with the indicated constructs, and proteins prepared $48 \mathrm{~h}$ later were subjected to immunoprecipitation using antibodies to c-myc followed by immunoblot analysis using antibodies to SUMO. Large arrowhead points to the singly sumoylated form of LIN-11; the small arrowhead points to the position of LIN-11 conjugated to two SUMO molecules. The doublets in lanes that were also transfected with HA-SUMO reveal that LIN-11 was sumoylated with both the endogenous and the exogenously expressed HA-SUMO. (Right) The same membrane which was reprobed with antibodies to myc. Position of nonsumoylated myc-LIN-11 is indicated by an arrow. $(B)$ Schematic representation of LIN-11. The putative SUMO acceptor sites (K17 and $\mathrm{K} 18$ ) and the additional lysines residues of LIN-11 are marked with asterisks. (C) In vitro sumoylation of LIN-11. myc-tagged wild-type LIN-11, or the indicated mutant forms were in vitro-transcribed and translated in the presence of ${ }^{35} \mathrm{~S}$-methionine and immunopurified with antibodies to myc. Protein G-bound LIN-11 was incubated with purified Aos1/Uba2 (E1), Ubc9 (E2), and SUMO-1 as indicated. Following extensive washes, protein G-bound material was separated on SDS-PAGE, stained, dried, and subjected to autoradiography. LIN-11 (thick arrow) and SUMO-modified LIN-11 (thin arrow) are indicated. $(D)$ Rescue of the vulva and utse phenotypes of lin-11(n389) transgenic animals harboring wild-type LIN-11 (plin-11::MYC::LIN-11) or mutant K17K18 LIN-11 (plin11::MYC:: LIN11 K17RK18R) transgenes. Each bar represents an independent transgenic line.
A

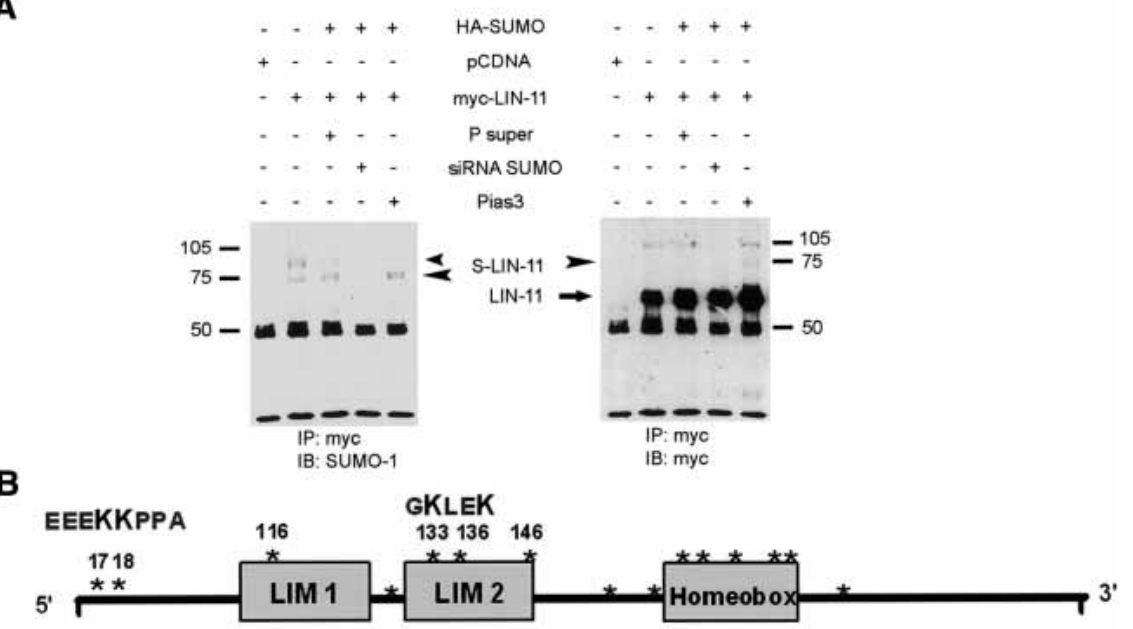

C
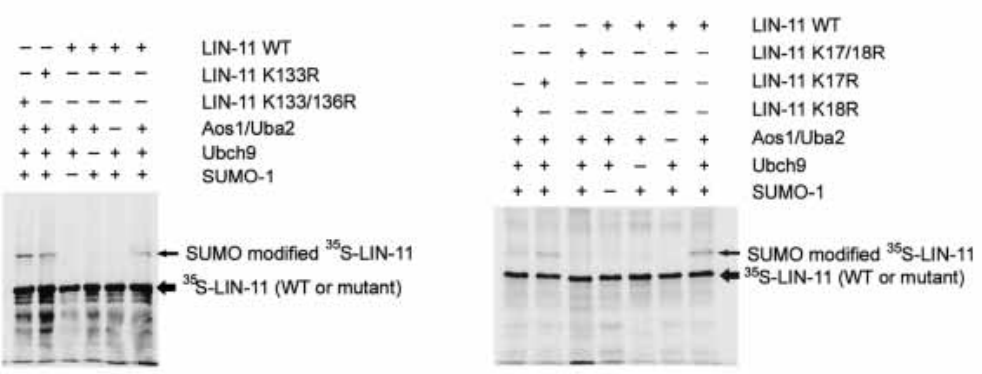

D
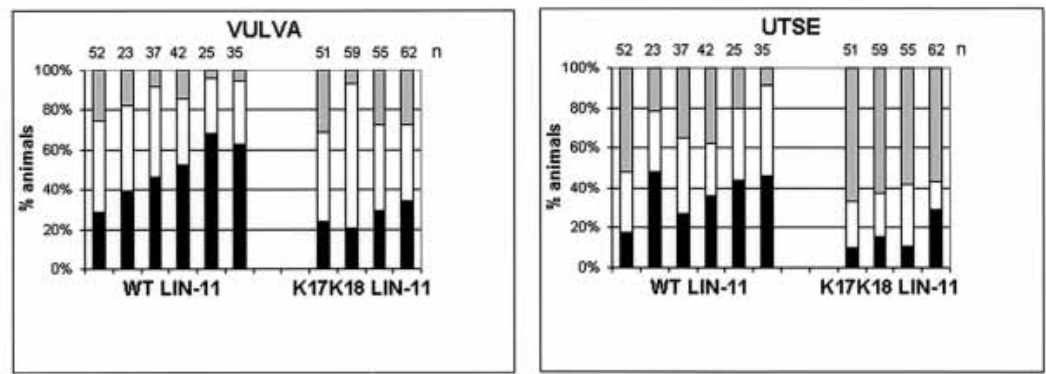

Black regions represent the proportion of animals displaying complete rescue, white regions represent the proportion of animals with partial rescue, and gray represents the proportion of transgenic animals in which the vulva or utse were not rescued. The average percentage of animals with nonrescued utse was $29.2 \% \pm 15.5 \%$ for the wild-type LIN-11 lines, and $61.0 \% \pm 4.6 \%$ for the mutant K17RK18R lines. The difference between these values is significant at $p<0.005$. Analysis was performed at the mid-L4 stage of synchronized populations. The number of animals analyzed from each line is indicated above each bar.

identified two LIN-11 species that differed in MW by about $20 \mathrm{kDa}$ (Fig. 7A, left panel). These observations suggest that LIN-11 is conjugated to two SUMO molecules. A noticeable level of LIN-11 sumoylation was also obtained when the SUMO E3 ligase PIAS3 (protein inhibitor of activated STAT3) (Nakagawa and Yokosawa 2002) was cotransfected (Fig. 7A). Additional confirmation for sumoylation of LIN-11 came from inhibition of SUMO expression using the siRNA SUMO construct, which inhibited sumoylation of LIN-11 (Fig. 7A, left panel). siRNA SUMO also efficiently reduced the in vivo sumoylation of c-Jun, which served as a positive control (Supplementary Fig. S4).

\section{K17 and K18 are the major SUMO acceptor sites in LIN-11 and are required for its uterine function}

To map the SUMO acceptor site(s) in LIN-11 (Fig. 7B), we first performed in vitro sumoylation of wild-type LIN-11. Full-length myc-LIN-11 was translated in vitro and purified (see Materials and Methods). Beads bound with ${ }^{35}$ S-labeled LIN-11 were then incubated with E1, E2 (SUMO-activating and SUMO-conjugating enzymes, respectively), and SUMO. The degree of sumoylation was followed by monitoring the formation of slower-migrating LIN-11 bands that were detected only upon addition of all components required for the sumoylation reaction (Fig. 7C). To identify the lysine(s) required for sumoyla- 
tion of LIN-11, we mutated different lysine residues at the $\mathrm{N}$-terminal region of LIN-11 and monitored possible changes in the degree of the in vitro sumoylation. K133 and K136 lie within a weak consensus motif for sumoylation (Fig. 7B; GKLE), but the level of LIN-11 sumoylation was not affected following mutagenesis of these sites (Fig. 7C, left panel). Additional mutagenesis of residues K116, K133, K136, and K146 to arginine individually or in combination did not affect sumolyation of LIN11 (data not shown). Only the LIN-11 construct containing lysine-to-arginine mutations at both $\mathrm{K} 17$ and $\mathrm{K} 18$ residues completely abolished sumoylation in vitro (Fig. 7C, right panel). Thus, both K17 and K18 can be modified by SUMO in vitro, although K18 appears to be the primary acceptor site.

To examine the physiological function of residues K17 and K18 of LIN-11, we generated transgenic lines in the lin-11(n389) background with either wild-type (plin$11::$ MYC $:: L I N-11 ; n=6$ ) or K17K18 mutated constructs (plin-11::MYC::LIN-11 K17RK18R; $n=4)$. The expression of a LIN-11 protein that cannot be sumoylated on $\mathrm{K} 17$ and K18 allowed us to examine the function of these residues in vivo. Analysis of these transgenic lines for rescue of the lin-11(n389) phenotypes demonstrated that although the level of the vulval rescue by the mutated construct was similar to that of the wild-type construct, rescue of utse formation was significantly weaker (Fig. 7D; Supplementary Fig. S5; for statistical analysis see Materials and Methods). These data suggest that residues $\mathrm{K} 17$ and K18 are required for LIN-11 activity mainly in the $\pi$ cells, to enable the formation of normal utse.

\section{Discussion}

The SUMO modification system has been widely studied in yeast and mammalian cells. However, the physiological function of the sumoylation process in organ development remained largely elusive. The present study provides the first analysis of postembryonic development of smo-1 deletion mutants. A maternal rescue effect allows completion of embryogenesis and postembryonic development of this mutant. Analysis of the developmental defects that characterize loss of SUMO function demonstrate that smo-1 is essential for the normal development of the reproductive system. In accordance, a strong expression of SMO-1::GFP is seen in the somatic gonad, vulva, hypodermis, and seam cells, in addition to its expression in the nervous system. This expression pattern together with the mutant phenotypes strongly suggests that there are many targets for sumoylation in C. elegans. For example, one of the signaling pathways implicated in the development of the gonad and germ line is Notch signaling (Greenwald 1998; Hubbard and Greenstein 2000). It is likely that members of the GLP-1 and LIN-12 signaling pathways may require SUMO modification for their activities. An additional example is the formation of pseudo-vulvae in the smo1(ok359) homozygous animals, which was restricted to the posterior side of the real vulva. This suggests that sumoylation of selective HOX family members or their upstream regulators is required for the control of the $C$. elegans posterior VPCs. A first line of support for this idea is the recent evidence that sumoylation of the PcG protein SOP-2 is required for its activity in the regulation of HOX genes (Zhang et al. 2004). However, some of the phenotypes observed in the smo-1 null animals can be attributed to a sequential developmental dependency type of effect. For example, lack of SUMO may primarily impair development of the somatic gonad, uterus, and spermatheca, which consequently results in aberrant formation of the germ line and vulva, the latter being a secondary event. This possibility is supported by laser ablation and molecular genetics experiments, which demonstrated that physiologic development of the germline and vulval tissues depends on signals from the somatic gonad (Kimble 1981; McCarter et al. 1997).

Our data show that in smo-1 deletion mutants the uterine $\pi$ cells were generated but failed to differentiate and to fuse to form the hymen between the vulva and the uterus (utse cell). The gonadal anchor cell also fails to fuse to the utse. Consequently, there is no connection between the vulva and the uterus. The specific defects found in $\pi$-cell differentiation resemble the uterine phenotypes of lin-11 mutants. On the basis of the similarities of the phenotypes, we hypothesized that LIN-11 may be a SUMO target. In smo-1 mutants the nuclear expression of LIN-11::GFP is reduced and shown to be diffuse. A similar pattern of diffuse expression was shown for the C. elegans SOP-2 mutant protein that was not properly sumoylated (Zhang et al. 2004).

Although we could detect expression of the LIN$11::$ GFP reporter in $s m o-1$ mutants, we could not detect myc expression in the smo-1 animals harboring the plin$11::$ MYC::LIN-11 transgene. The differences between these constructs are the use of the truncated form of LIN-11 in the LIN-11::GFP construct (Hobert et al. 1998) and its fusion to the stable GFP reporter, which probably enabled the accumulation of the reporter to detectable levels.

Expression of the fusion protein plin-11::MYC::SMO$1::$ LIN-11 localized the protein to distinct nuclear bodies and also partially rescued $\pi$-cell differentiation in the smo-1(ok359) homozygous animals, but had a negative effect on late stages of vulval morphogenesis. Several SUMO-modified proteins have been observed to localize to distinct subnuclear structures such as PML, Sp3, and LEF-1 (Zhong et al. 2000; Sachdev et al. 2001; Ross et al. 2002). The fact that these nuclear bodies are implicated in transcription regulation (Zhong et al. 2000) suggests that modification of LIN-11 by SUMO and its translocation to the nuclear bodies regulate its transcriptional activity. The opposite effects of SUMO-modified LIN-11 on the development of the uterus and vulva in smo1(ok359) homozygous animals suggest that SUMO modification may activate or repress LIN-11, depending on the organ and the nature of the protein complex associated with LIN-11.

Our data obtained using both in vivo and in vitro assays show that LIN-11 is a substrate for SUMO modification. However, we could not detect SUMO-modified 
LIN-11 following immunoprecipitation of LIN-11 from worm extracts (transgenic lines that express myc-tagged lin-11 cDNA under the regulation of lin-11 upstream and 3' UTR sequences). The possible reasons are the small number of cells that express LIN-11 (Hobert et al. 1998; Newman et al. 1999; Gupta et al. 2003), the small fraction of LIN-11 that may be modified by SUMO, and the dynamic and reversible nature of this modification.

We found that mutating a weak consensus motif for SUMO conjugation on LIN-11 (GKLEK) was not sufficient to abolish its sumoylation, whereas mutagenesis of the N-terminal K17K18 abolished LIN-11 sumoylation. We assayed the functional importance of K17K18 by performing transgenic rescue experiments of lin-11(n389) with LIN-11 mutated on K17K18. This experiment demonstrated the requirement of these two residues for the function of LIN-11 in the $\pi$ cells. While the UTSE was not rescued in the majority of the animals that express the mutant construct $(61 \%)$, a partial rescue was observed and could be attributed to overexpression of LIN11 in the extrachromosomal array. High level of the nonsumolyated mutant LIN-11 may alter its subcellular distribution and force its presence within the transcriptional complex. Although K17K18 are also potential sites for other posttranslational modification, our data provide strong evidence for the role of sumolyation in the regulation of LIN-11 activity.

Overall, through the characterization of a genetically deleted smo-1 strain of C. elegans, the current study identifies a critical function of SUMO during the development of the reproductive system, and has identified LIN-11 as a new substrate whose sumoylation is associated with the formation of a functional vulval-uterine connection, an essential structure of the egg-laying system in C. elegans.

\section{Materials and methods}

\section{Strains and alleles}

Standard conditions for culturing C. elegans were used (Brenner 1974). All experiments were performed at $20^{\circ} \mathrm{C}$ unless otherwise indicated. The wild-type parent for the strains used in this study is the C. elegans var. Bristol strain N2. The relevant genes and alleles used in this study are: LGI: smo-1(ok359) (isolated and kindly provided by the C. elegans Gene Knockout Project team at Oklahoma Medical Research Foundation [OMRF]) and lin-11(n389) (Ferguson and Horvitz 1985). In addition, the following integrated transgenes or extrachromosomal arrays were used: jcIs1[ajm-1::GFP] (Mohler et al. 1998; Köppen et al. 2001), syIs49[zmp-1::GFP; dpy-20(+)] (Wang and Sternberg 2000), kuIs29[cog-2::GFP, unc-119(+)] (Hanna-Rose and Han 1999), [lin-11(n389); kuIs29], mgIs21[plin-11-ABCDE:: GFP], a translational fusion including the first 200 amino acids from the N-terminal region of LIN-11 fused to GFP (Hobert et al. 1998), hyEx80[SMO-1::GFP; rol-6] (this study), hyEx81 [plin-11::MYC::LIN-11; rol-6] (this study), hyEx82/plin-11:: MYC::SMO-1::LIN-11; rol-6] (this study), Ex/plin-11::MYC:: LIN-11; myo-2::GFP, unc-119(+)] (this study), Ex/plin-11:: MYC::LIN-11 K17RK18R; myo-2::GFP, unc-119(+)] (this study).

\section{Genetic analysis of the smo-1 deletion mutant}

The deletion allele smo-1(ok359) was isolated by the C. elegans Gene Knockout Project team at OMRF using a PCR screen of a mutant collection of TMP/UV mutagenized animals. The strain was outcrossed 10 times before analysis.

Sterility in smo-1(ok359) homozygous at $20^{\circ} \mathrm{C}$ was assayed by plating out all self progeny from smo-1(ok359)/+ hermaphrodites and scoring the animals for protruded vulva $(\mathrm{Pvl})$ and sterile phenotypes (Ste). All self-progeny were viable, and 23.6\% $(n=919)$ were found to be Pvl and sterile. No dead embryos or larvae were found on plates. No self-progeny were produced by a hermaphrodite homozygous for smo-1(ok359) (0\%, $n>1000)$. At $15^{\circ} \mathrm{C}, 19.7 \%$ of progeny $(n=426)$ were found to be Pvl and sterile, which may indicate low penetrance lethality during embryogenesis or earlier arrest during oogenesis and/or spermatogenesis. At $25^{\circ} \mathrm{C}$, the self-progeny of smo-1 (ok359) heterozygous included only $5 \%$ sterile (not Pvl) animals. The progeny mean size was $85 \pm 39$ (self-progeny of seven hermaphrodites were analyzed; $n=598$ ), suggesting that this allele is temperaturesensitive and that elevated temperature caused arrest during oogenesis and/or spermatogenesis.

Analysis of homozygous smo-1(ok359) males indicated that the male tail failed to develop normally, as reflected in abnormal structure of the rays, resulting in its inability to mate (data not shown).

Rescue analysis to verify the genetic deletion was performed by the generation of transgenic strains containing the smo-1 genomic region. The psmo.g1 plasmid (see below) was injected at $1 \mu \mathrm{g} / \mathrm{mL}$ together with $80 \mu \mathrm{g} / \mathrm{mL}$ of the transformation marker pRF4 (rol-6) into smo-1(ok359) heterozygous. Individual animals from stable lines were isolated to obtain transgenic homozygous lines. Positive rescuing activity was scored as homozygous smo-1(ok359) transgenic fertile adults with no Pvl phenotype, and normal egg-laying behavior. Six independent viable transgenic lines were obtained, and all of them rescued the homozygous phenotype of smo-1(ok359) animals.

\section{Plasmid and PCR-amplified constructs}

For the rescue experiments, the PCR product was amplified from C. elegans genomic DNA with the primers K12C11A1 (5'-GTCGAAAACACTGGAAATTGCTGT-3') and K12C11OL (5'-TCCTCGTCAAATCCGAAATC-3') and cloned into the pTOPO vector (Invitrogen) to create psmo.g1. The cloned genomic region in the plasmid psmo.gl contains $1 \mathrm{~Kb}$ upstream of the smo-1 gene (until the start of the upstream gene K12C11.3), the entire smo-1 gene $(\mathrm{K} 12 \mathrm{C} 11.2)$, and $0.5 \mathrm{~Kb}$ of the $3^{\prime}$ UTR (in the last exon of the downstream gene K12C11.1) (Fig. 1D).

The SMO-1::GFP reporter is a PCR product (generated according to Hobert et al. 1999) amplified from C. elegans genomic DNA with the primers K12C11A1 (5'-GTCGAAAA CACTGGAAATTGCTGT- $\left.3^{\prime}\right)$ and K12C11B (5'-AGTCGACC TGCAGGCATCCAAGCTTGAATCCGCCCAGCTGCTCTTG GTA-3') and then reamplified with internal primers and a GFP fragment of pPD95.75 (kindly provided by A. Fire, Stanford University School of Medicine, Stanford, CA) to create a GFP fusion construct. The final product contains $1 \mathrm{~Kb}$ upstream of the smo- 1 coding region, the entire coding region fused to GFP, and the 3' UTR of unc- 54. Three transgenic lines of the translational fusion were analyzed.

The full-length lin-11 cDNA construct was generated by PCR using the partial lin-11 cDNA in the vector pYK452F7-3 (Gupta and Sternberg 2002). A myc tag was added upstream to the lin11 cDNA, and the construct was shuttled into the pCDNA plasmid for the sumolyation and RNAi assays. The primers 
used were CD5 (include the first seven amino acids of LIN-11) (5'-GGGGAAGATCTCATTCTTCTTCTTCGTTCATCATCAC CTCA-3') and CD3 (5'-GGCCGCTCGAGCTACCATGAAA CCGGAGTTGG-3').

The plin-11::MYC::SMO-1::LIN-11 fusion protein was generated by cloning the $3.5-\mathrm{Kb} 5^{\prime}$ sequences of 1 in- 11 genomic region upstream of the myc peptide followed by the genomic sequence of smo-1 (which includes amino acids 1-88). The Cterminal three amino acids of the full-length SMO-1, including the Gly-Gly found at the C-terminal of mature SUMO, were not included in order to prevent cleavage of the fusion protein by C-terminal SUMO hydrolases. The lin-11 cDNA followed by $1.7-\mathrm{Kb} 3^{\prime}$ UTR sequences of the lin-11 genomic region were cloned downstream of the smo-1 sequence. The control construct for this experiment and for the rescue experiments of lin-11(n389), plin-11::MYC::LIN-11, was generated by cloning of the $5^{\prime} 3.5-\mathrm{Kb}$ region of lin-11 upstream of myc followed by the lin-11 cDNA and the 1.7-Kb 3' UTR of lin-11 genomic region. Site-directed mutagenesis of this construct on amino acids 17 and 18 (lysine to arginine) was performed using the PCR method. All constructs were verified by sequencing.

\section{Analysis of the LIN-11 K17RK18R transgenic lines and statistics}

Analysis of the Ex/plin-11::MYC::LIN-11; myo-2::GFP, unc119(+)] and Ex[plin-11::MYC::LIN-11 K17RK18R; myo-2:: GFP, unc-119(+)] transgenic lines for their ability to rescue lin11(n389); unc-119(ed4) was performed on L4 synchronized populations. Only non-unc, myo-2::GFP animals were scored. unc animals that did not express the GFP reporter were used as negative controls.

ANOVA was used to compare the two groups of the lin11(n389) transgenic lines (Fig. 7D). The null hypothesis was that no difference exists between the vulva/utse and egg-laying ability of the wild-type and mutant lines. In the vulva assay, there was moderate evidence of a difference between wild-type and mutant transgenes in the proportion of animals with complete rescue $(p<0.05)$. In the utse assay, moderate evidence of a difference was found for both complete and partial rescue $(p<0.05)$. Very strong evidence of a difference between the wildtype and mutant lines was observed in the proportion of animals in which the utse was not rescued (Fig. 7D, gray region; $p<0.005$ ).

\section{Immunofluorescence and confocal analysis}

Immunofluorescence of whole-mount animals was performed with the monoclonal antibody MH27 (kindly provided by M. Hresko [University of Washington School of Medicine, Seattle, WA] and R. Waterston [University of Washington, School of Medicine, Seattle, WA]; Podbilewicz and White 1994). Larvae and adults were fixed and stained according to Finney (Finney and Ruvkun 1990; Sharma-Kishore et al. 1999). Gonad dissection and staining with the SP56 monoclonal antibody was done as described in Kadyk and Kimble (1998). Confocal microscopy images were captured as a stacked series using a Bio-Rad MRC 1024 confocal scanning microscope and processed using Adobe Photoshop.

\section{In vitro sumoylation assay}

In vitro translated ${ }^{35} \mathrm{~S}$-LIN-11 (TNT, Promega) served as the substrate for in vitro reaction, following its immunopurification from reticulocyte lysates with the aid of antibodies to myc (LIN11 is myc-tagged). Immunopurified LIN-11 was coupled to pro-
tein-G beads, which were extensively washed prior to the addition of purified Aos1/Uba2 (15 ng), and Ubc9 $(0.5 \mu \mathrm{g})$. The sumoylation reaction was carried out for $90 \mathrm{~min}$ at $37^{\circ} \mathrm{C}$ in conjugation buffer (20 mM HEPES at $\mathrm{pH} 7.4,5 \mathrm{mM} \mathrm{MgCl}_{2}, 2$ mM ATP, $10 \mathrm{mM}$ creatine phosphate, 1 unit of creatine phosphokinase). Bead-bound complexes were washed $(2 \times 0.5 \mathrm{M} \mathrm{LiCl})$ before denaturating for $5 \mathrm{~min}$ at $95^{\circ} \mathrm{C}$ in $3 \times$ sample buffer. Proteins were separated on $8 \%$ SDS-PAGE. Gels were stained with Coomassie Blue, dried, and exposed to X-ray film (X-Omat, Eastman Kodak). Mutations of the indicated lysine residues of LIN11 were generated by a PCR method and verified by sequencing.

\section{In vivo sumoylation assays}

$293 \mathrm{~T}$ cells $\left(5 \times 10^{6}\right)$ were transfected with the indicated plasmids $(3 \mu \mathrm{g})$ and cells were harvested $(48 \mathrm{~h})$ in PBS containing 20 mM N-ethylmaleimide (Sigma). Cells were then resuspended in RIPA buffer $(50 \mathrm{mM}$ Tris- $\mathrm{HCl}, 150 \mathrm{mM} \mathrm{NaCl}, 0.5 \%$ sodium deoxycholate, $0.1 \%$ SDS, $1 \%$ Nonidet P-40, $1 \mathrm{mM}$ dithiothreitol, protease inhibitor cocktail, $25 \mathrm{mM} \mathrm{N}$-ethylmaleimide, and $200 \mu \mathrm{M}$ iodoacetamide), and subjected to sonication (30 sec on ice). Protein extracts $(1.2 \mathrm{mg})$ were precleared with protein-G beads (Invitrogen) and incubated (overnight at $4^{\circ} \mathrm{C}$ ) with antibodies (1 $\mu \mathrm{g} / \mathrm{mg}$ ) to myc (9E10) or to c-Jun (Santa Cruz). Immune complexes were captured on protein-G beads which were then washed with RIPA buffer before being subjected to SDS-PAGE $(8 \%)$, followed by electroblotting to membrane which was blocked ( $5 \%$ nonfat dry milk) and incubation with monoclonal antibodies against GMP-1 (SUMO-1) (Zymed Laboratories), cmyc, or c-Jun $\left(1: 1000\right.$; overnight at $\left.4^{\circ} \mathrm{C}\right)$. Membranes were washed and incubated with secondary antibodies coupled to horseradish peroxidase before development with the aid of chemiluminescence.

\section{Acknowledgments}

We thank the C. elegans Gene Knockout Project team at OMRF for generating the smo-1 null strain. We thank the Caernorhabditis Genetics Center (CGC) for providing strains, Reuven Agami for the kind gift of the pSUPER construct, Ke Shuai for the PIAS3 plasmid, Susan Strome for the SP56 antibody, and Chelly Hresko and Bob Waterston for the MH27 antibody. We also thank the members of the Ronai and Podbilewicz Labs for discussions. P.W.S. is an Investigator and P.B.G. was an Associate with the HHMI. Support from NCI grant CA59908 (to Z.R.), HFSP, and the Israel Science Foundation (to B.P.) is gratefully acknowledged.

\section{References}

Alarcon-Vargas, D. and Ronai, Z. 2002. Sumo in cancer-Wrestlers wanted. Cancer Biol. Ther. 1: 237-242.

Brenner, S. 1974. The genetics of Caenorhabditis elegans. Genetics 77: 71-94.

The C. elegans Sequencing Consortium. 1998. Genome sequence of the nematode C. elegans: A platform for investigating biology. The C. elegans Sequencing Consortium. Science 282: 2012-2018.

Choudhury, B.K. and Li, S.S. 1997. Identification and characterization of the SMT3 cDNA and gene from nematode Caenorhabditis elegans. Biochem. Biophys. Res. Comm. 234: 788-791.

Coulson, A. 1996. The Caenorhabditis elegans genome project. C. elegans Genome Consortium. Biochem. Soc. Trans. 24: 289-291. 
Epps, J.L. and Tanda, S. 1998. The Drosophila semushi mutation blocks nuclear import of bicoid during embryogenesis. Curr. Biol. 8: 1277-1280.

Ferguson, E.L. and Horvitz, H.R. 1985. Identification and characterization of 22 genes that affect the vulval cell lineages of the nematode Caenorhabditis elegans. Genetics 110: $17-72$.

Finney, M. and Ruvkun, G. 1990. The unc-86 gene product couples cell lineage and cell identity in C. elegans. Cell 63: 895-905.

Francis, R. and Waterston, R.H. 1991. Muscle cell attachment in Caenorhabditis elegans. J. Cell. Biol. 114: 465-479.

Fraser, A.G., Kamath, R.S., Zipperlen, P., Martinez-Campos, M., Sohrmann, M., and Ahringer, J. 2000. Functional genomic analysis of $C$. elegans chromosome I by systematic RNA interference. Nature 408: 325-330.

Freyd, G., Kim, S.K., and Horvitz, H.R. 1990. Novel cysteinerich motif and homeodomain in the product of the Caenorhabditis elegans cell lineage gene lin-11. Nature 344: 876-879.

Gostissa, M., Hengstermann, A., Fogal, V., Sandy, P., Schwarz, S.E., Scheffner, M., and Del Sal, G. 1999. Activation of p53 by conjugation to the ubiquitin-like protein SUMO-1. EMBO J. 18: 6462-6471.

Greenwald, I. 1998. LIN-12/Notch signaling: Lessons from worms and flies. Genes \& Dev. 12: 1751-1762.

Gupta, B.P. and Sternberg, P.W. 2002. Tissue-specific regulation of the LIM homeobox gene lin-11 during development of the Caenorhabditis elegans egg-laying system. Dev. Biol. 247: 102-115.

Gupta, B.P., Wang, M., and Sternberg, P.W. 2003. The C. elegans LIM homeobox gene lin-11 specifies multiple cell fates during vulval development. Development 130: 2589-2601.

Hanna-Rose, W. and Han, M. 1999. COG-2, a sox domain protein necessary for establishing a functional vulval-uterine connection in Caenorhabditis elegans. Development 126: 169-179.

Hobert, O., D'Alberti, T., Liu, Y., and Ruvkun, G. 1998. Control of neural development and function in a thermoregulatory network by the LIM homeobox gene lin-11. J. Neurosci. 18: 2084-2096.

Hobert, O., Moerman, D.G., Clark, K.A., Beckerle, M.C., and Ruvkun, G. 1999. A conserved LIM protein that affects muscular adherens junction integrity and mechanosensory function in Caenorhabditis elegans. J. Cell. Biol. 144: $45-57$.

Hubbard, E.J. and Greenstein, D. 2000. The Caenorhabditis elegans gonad: A test tube for cell and developmental biology. Dev. Dyn. 218: 2-22.

Johnson, E.S. and Blobel, G. 1999. Cell cycle-regulated attachment of the ubiquitin-related protein SUMO to the yeast septins. J. Cell. Biol. 147: 981-993.

Jones, D., Crowe, E., Stevens, T.A., and Candido, E.P. 2002. Functional and phylogenetic analysis of the ubiquitylation system in Caenorhabditis elegans: Ubiquitin-conjugating enzymes, ubiquitin-activating enzymes, and ubiquitin-like proteins. Genome Biol. 3: 0002.1-0002.15.

Kadyak, L.C. and Kimble, J. 1998. Genetic regulation of entry into meiosis in Caenorhabditis elegans. Development 125: $1803-1813$.

Kelly, W.G. and Fire, A. 1998. Chromatin silencing and the maintenance of a functional germline in Caenorhabditis elegans. Development 125: 2451-2456.

Kimble, J. 1981. Alterations in cell lineage following laser ablation of cells in the somatic gonad of Caenorhabditis elegans. Dev. Biol. 87: 286-300.
Kirsh, O., Seeler, J.S., Pichler, A., Gast, A., Muller, S., Misaka, E., Mathieu, M., Harel-Bellan, A., Kouzarides, T., Melchoir, F., et al. 2002. The SUMO E3 ligase RanBP2 promotes modification of the HDAC4 deacetylase. EMBO I. 21: 26822691.

Köppen, M., Simske, J.S., Sims, P.A., Firestein, B.L., Hall, D.H., Radice, A.D., Rongo, C., and Hardin, J.D. 2001. Cooperative regulation of AJM-1 controls junctional integrity in Caenorhabditis elegans epithelia. Nat. Cell Biol. 3: 983-991.

Kurepa, J., Walker, J.M., Smalle, J., Gosink, M.M., Davis, S.J., Durham, T.L., Sung, D-Y., and Vierstra, R.D. 2002. The SUMO protein modification system in Arabidopsis: accumulation of SUMO1 and 2 conjugates is increased by stress. J. Biol. Chem. 278: 6862-6872.

Le Drean, Y., Mincheneau, N., Le Goff, P., and Michel, D. 2002. Potentiation of glucocorticoid receptor transcriptional activity by sumoylation. Endocrinology 143: 3482-3489.

Mahajan, R., Delphin, C., Guan, T., Gerace, L., and Melchoir, F. 1997. A small ubiquitin-related polypeptide involved in targeting RanGAP1 to nuclear pore complex protein RanBP2. Cell 88: 97-107.

Matunis, M.J., Coutavas, E., and Blobel, G. 1996. A novel ubiquitin-like modification modulates the partitioning of the Ran-GTPase-activating protein RanGAP1 between the cytosol and the nuclear pore complex. J. Cell Biol. 135: 14571470.

McCarter, J., Bartlett, B., Dang, T., and Schedl, T. 1997. Somagerm cell interactions in Caenorhabditis elegans: Multiple events of hermaphrodite germline development require the somatic sheath and spermathecal lineages. Dev. Biol. 181: 121-143.

Melchoir, F. 2000. SUMO-neoclassical ubiquitin. Ann. Rev. Cell Dev. Biol. 16: 591-626.

Mohler, W.A., Simske, J.S., Williams-Masson, E.M., Hardin, J.D., and White, J.G. 1998. Dynamics and ultrastructure of developmental cell fusions in the Caenorhabditis elegans hypodermis. Curr. Biol. 8: 1087-1090.

Müller, S., Matunis, M.J., and Dejean, A. 1998. Conjugation with the ubiquitin-related modifier SUMO-1 regulates the partitioning of PML within the nucleus. EMBO J. 17: 61-70.

Müller, S., Berger, M., Lehembre, F., Seeler, J.S., Haupt, Y., and Dejean, A. 2000. C-Jun and p53 activity is modulated by SUMO-1 modification. J. Biol. Chem. 275: 13321-13329.

Müller, S., Ledl, A., and Schmidt, D. 2004. SUMO: A regulator of gene expression and genome integrity. Oncogene 23: $1998-2008$.

Nakagawa, K. and Yokosawa, H. 2002. PIAS3 induces SUMO-1 modification and transcriptional repression of IRF-1. FEBS Lett. 530: 204-208.

Newman, A.P. and Sternberg, P.W. 1996. Coordinated morphogenesis of epithelia during development of the Caenorhabditis elegans uterine-vulval connection. Proc. Natl. Acad. Sci. 93: 9329-9333.

Newman, A.P., White, J.G., and Sternberg, P.W. 1995. The Caenorhabditis elegans lin-12 gene mediates induction of ventral uterine specialization by the anchor cell. Development 121: 263-271.

-1996. Morphogenesis of the C. elegans hermaphrodite uterus. Development 122: 3617-3626.

Newman, A.P., Acton, G.Z., Hartwieg, E., Horvitz, H.R., and Sternberg, P.W. 1999. The lin-11 LIM domain transcription factor is necessary for morphogenesis of C. elegans uterine cells. Development 126: 5319-5326.

Pichler, A., Gast, A., Seeler, J.S., Dejean, A., and Melchior, F. 2002. The nucleoporin RanBP2 has SUMO1 E3 ligase activity. Cell 108: 109-120. 
Podbilewicz, B. and White, J.G. 1994. Cell fusions in the developing epithelial of C. elegans. Dev. Biol. 161: 408-424.

Poukka, H., Karvonen, U., Janne, O.A., and Palvimo, J.J. 2000. Covalent modifications of the androgen receptor by small ubiquitin-like modifier 1 (SUMO-1). Proc. Nat1. Acad. Sci. 97: 14145-14150.

Ross, S., Best, J.L., Zon, L.I., and Gill, G. 2002. SUMO-1 modification represses $\mathrm{Sp} 3$ transcriptional activation and modulates its subnuclear localization. Mol. Cell 10: 831-842.

Sachdev, S., Bruhn, L., Sieber, H., Pichler, A., Melchior, F., and Grosschedl, R. 2001. PIASy, a nuclear matrix-associated SUMO E3 ligase, represses LEF1 activity by sequestration into nuclear bodies. Gene \& Dev. 15: 3088-3103.

Schmidt, D. and Muller, S. 2002. Members of the PIAS family act as SUMO ligases for c-Jun and p53 and repress p53 activity. Proc. Nat1. Acad. Sci. 99: 2872-2877.

Schwarz, S.E., Matuschewski, K., Liakopoulos, D., Scheffner, M., and Jentsch, S. 1998. The ubiquitin-like proteins SMT3 and SUMO-1 are conjugated by the UBC9 E2 enzyme. Proc. Natl. Acad. Sci. 95: 560-564.

Sharma-Kishore, R., White, J.G., Southgate, E., and Podbilewicz, B. 1999. Formation of the vulva in Caernorhabditis elegans: A paradigm for organogenesis. Development 126: 691-699.

Steffan, J.S., Agrawal, N., Pallos, J., Rockabrand, E., Trotman, L.C., Slepko, N., Illes, K., Lukacsovich, T., Zhu, Y-Z., Cattaneo, E., et al. 2004. SUMO modification of Huntingtin and Huntington's disease pathology. Science 304: 100-104.

Sternsdorf, T., Jensen, K., and Will, H. 1997. Evidence for covalent modification of the nuclear dot-associated proteins PML and Sp100 by PIC1/SUMO-1. J. Cell Biol. 139: 1621-1634.

Wang, M. and Sternberg, P.W. 2000. Patterning of the C. elegans $1^{0}$ vulval lineage by RAS and Wnt pathways. Development 127: 5047-5058.

Ward, S., Roberts, T.M., Strome, S., Pavalko, F.M., and Hogan, E. 1986. Monoclonal antibodies that recognize a polypepetide antigenic determinant shared by multiple Caenorhabditis elegans sperm-specific proteins. J. Cell. Biol. 102: 1778-1786.

Zhang, H., Smolen, G.A., Palmer, R., Christoforou, A., van den Heuvel, S., and Haber, D.A. 2004. SUMO modification is required for in vivo HOX gene regulation by the Caenorhabditis elegans Polycomb group protein SOP-2. Nat. Genet. 36: $507-511$.

Zhong, S., Salomoni, P., and Pandolfi, P.P. 2000. The transcriptional role of PML and the nuclear body. Nat. Cell Biol. 2: E85-E90. 


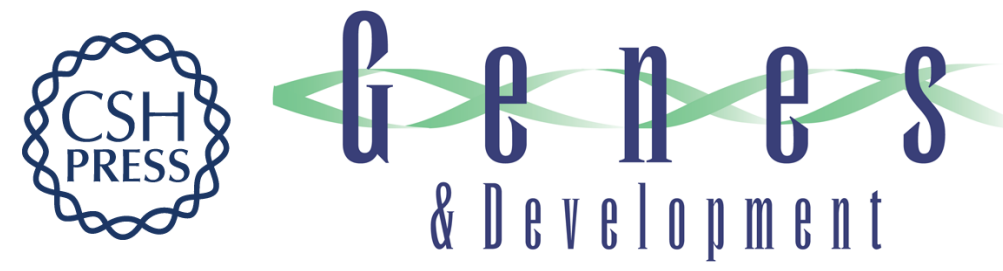

\section{The small ubiquitin-like modifier (SUMO) is required for gonadal and uterine-vulval morphogenesis in Caenorhabditis elegans}

Limor Broday, Irina Kolotuev, Christine Didier, et al.

Genes Dev. 2004, 18:

Access the most recent version at doi:10.1101/gad.1227104

Supplemental http://genesdev.cshlp.org/content/suppl/2004/10/06/18.19.2380.DC1
Material

References This article cites 54 articles, 31 of which can be accessed free at:

http://genesdev.cshlp.org/content/18/19/2380.full.html\#ref-list-1

License

Email Alerting Receive free email alerts when new articles cite this article - sign up in the box at the top

Service right corner of the article or click here.

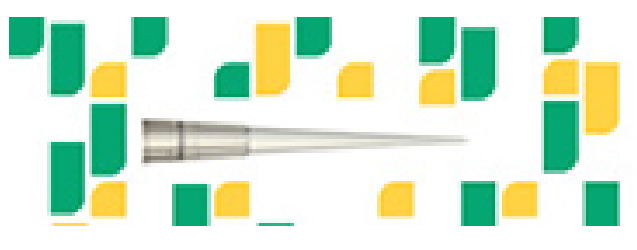

Focused on your science. 